\title{
EVALUATING A DE-CLUTTERING TECHNIQUE FOR NEXTGEN RNAV AND RNP CHARTS
}

\author{
Abhizna Butchibabu ${ }^{*}$, Rebecca Grayhem ${ }^{* *}$, R. John Hansman ${ }^{*} \&$ Divya Chandra ${ }^{* *}$ \\ *Massachusetts Institute of Technology, Cambridge, MA \\ ${ }^{* *}$ US DOT Volpe Center, Cambridge, MA
}

\begin{abstract}
The authors propose a de-cluttering technique to simplify the depiction of visually complex Area Navigation (RNAV) and Required Navigation Performance (RNP) procedures by reducing the number of paths shown on a single chart page. An experiment was conducted to determine whether charts with fewer paths ("Modified charts") allow improved access to information in terms of time and accuracy compared with charts that are currently used ("Current charts").

Data were collected from 28 airline and 19 corporate pilots. Results show that pilot response times were significantly improved with the Modified charts. For approach procedures, the mean response time was 16.9 seconds for Current charts and 10.7 seconds for Modified charts. For departure procedures, the mean response time was 16.2 seconds for Current charts and 13.2 seconds for Modified charts. This difference in response time between Current and Modified charts was consistent across the different procedures (approach and departure), pilot types (Airline and Corporate), and chart manufacturers (FAA and Jeppesen) included in the study. Additionally, pilots answered questions correctly $99.5 \%$ of the time with no difference in response accuracy between Current and Modified charts. Note, this experiment only evaluated the potential benefit of separating paths across multiple pages and did not explore the drawbacks to this decluttering technique.
\end{abstract}

\section{Introduction and Motivation}

The Federal Aviation Administration (FAA) and International Civil Aviation Organization (ICAO) are transitioning to Performance-Based Navigation (PBN) to increase National Airspace System (NAS) capacity and efficiency. PBN is a key component of the FAA's Next Generation Air Transportation System (NextGen). PBN routes and procedures, which include Area Navigation (RNAV) and Required Navigation Performance (RNP), are being developed to facilitate this transition [1]. RNAV and RNP procedures are designed to take advantage of advanced navigation technology. RNAV enables aircraft to fly directly from point-to-point on any desired flight path using ground- or spaced-based navigation aids. RNP is RNAV with the addition of onboard monitoring and alerting capability. RNP procedures meet specific requirements for position determination and track conformance, enabling the aircraft to fly accurate routes without flying directly over ground-based navigation aids. Both RNAV and RNP offer operators safety improvements, more flexibility to negotiate terrain, increased airspace capacity and enhanced operational efficiency.

Several human factors issues with RNAV and RNP procedures have emerged, including procedure complexity, chart clutter, and nonconformance with altitude constraints [2], [3], [4]. Studies of Aviation Safety Reporting System (ASRS) reports [2], [3] identified approximately $30 \%$ of 124 ASRS reports from seven specific airports that were related to procedure design. Another study [4] found approximately 59 out of 285 ASRS reports (21\%) were due to chart and procedure design with chart clutter, procedure complexity, and waypoint confusion identified as factors.

The authors reviewed approximately 150 RNAV arrival and departure and RNAV (RNP) approach procedure charts [5], [6] and categorized them as either "Problematic" or "Baseline." Problematic procedures were identified based on operational safety reports obtained through ASRS and subject matter experts. Baseline procedures consisted of the top 35 airports included in the Operational Evolution Plan (OEP) that were not mentioned in the ASRS reports or by subject matter experts. Procedure variables (e.g., number of waypoints, number of altitude constraints, and length of path segments) 
were compared between the Problematic and Baseline procedures.

Results from the chart review suggest that the Problematic RNAV (RNP) approach and RNAV departure procedures contain more paths than the Baseline group. The authors concluded that depicting multiple paths on a chart increases visual clutter and information density [5], [6], potentially making these charts more difficult to use.

One way to mitigate the potential adverse effects of multiple paths per chart is to reduce the number of paths depicted on a single chart. This can be done by separating paths onto different chart pages. An example of this technique is shown for an approach procedure into Boise, Idaho airport in Figure 1 (Current chart) and Figure 2 (Modified chart). An example for a departure procedure into Salt Lake City, Utah is shown in Figure 3 (Current) and Figure 4 (Modified). The advantage of this method is that it simplifies the chart by depicting less information and reducing visual clutter, which might improve a pilot's ability to retrieve information from the chart.

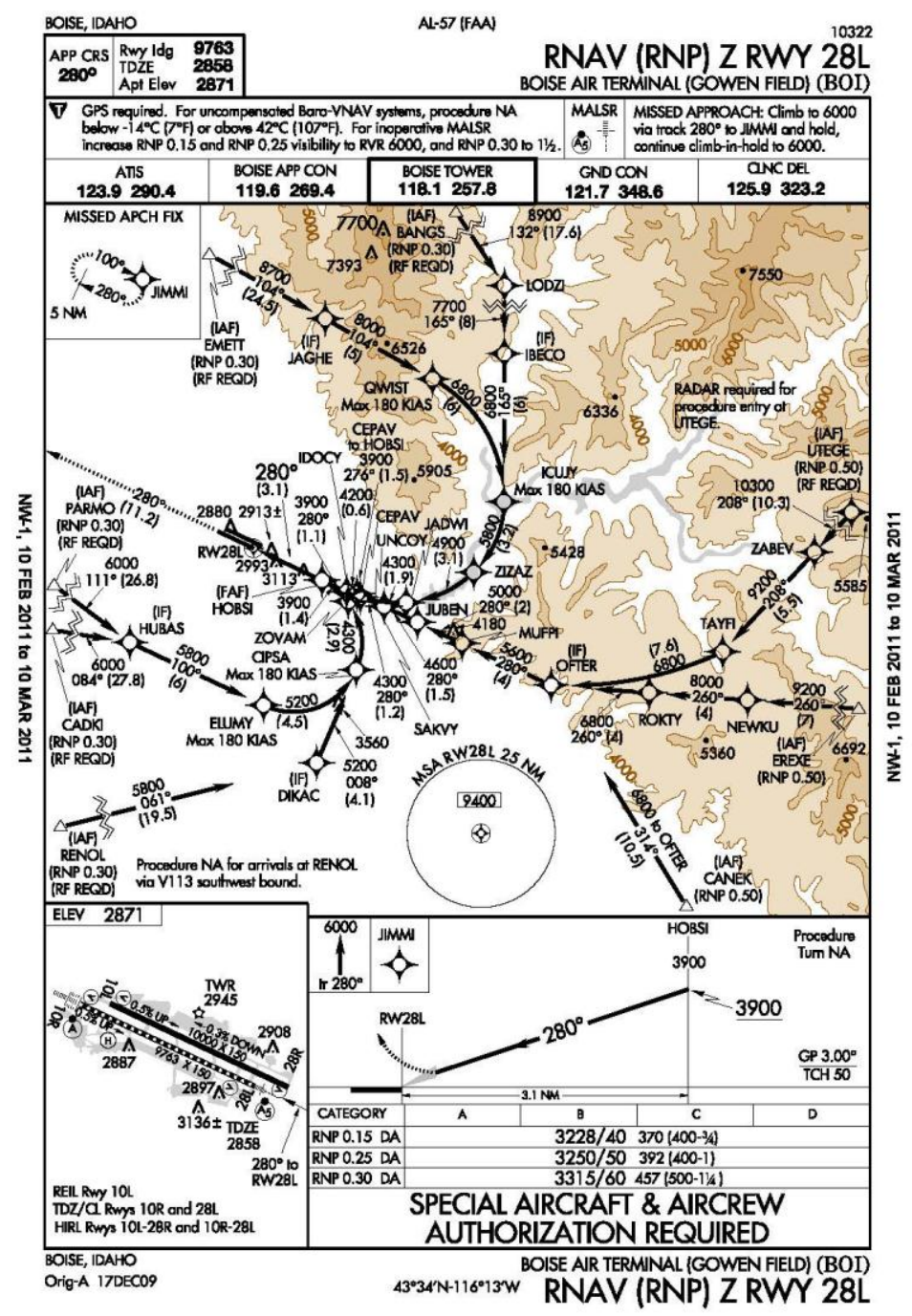

Figure 1. Example Current RNAV (RNP) Approach Chart 


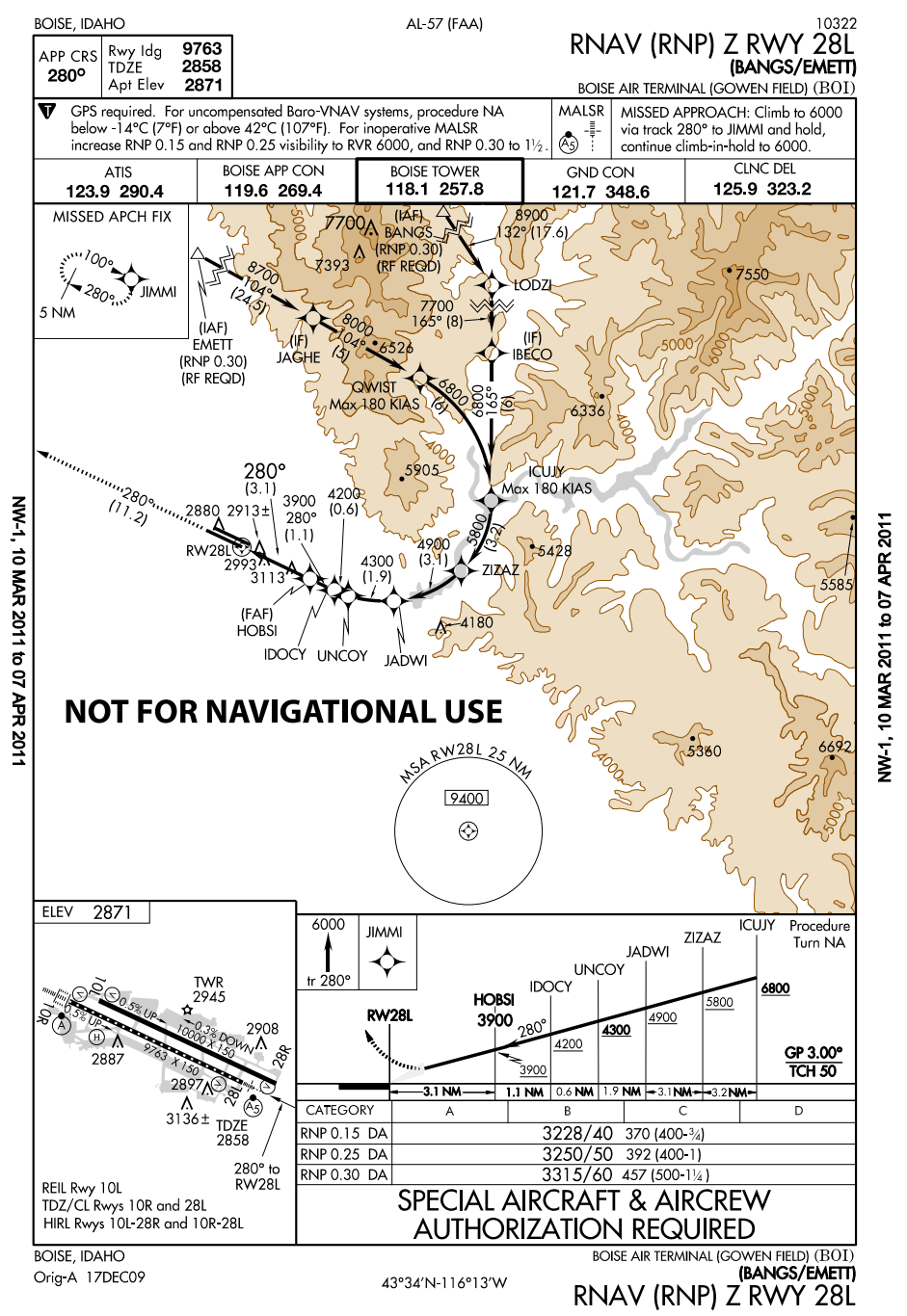

Figure 2. Example Modified RNAV (RNP) Approach Chart

Another problem related to depicting multiple paths on RNAV (RNP) approach procedures is the difficulty of depicting the full vertical profile for each path [7]. Each lateral path may have a different associated vertical path and it is difficult to clearly depict multiple profiles in one profile view. The current practice is to depict only the part of the vertical profile that begins at either the Intermediate Fix (IF) or the Final Approach Fix (FAF) that all the paths have in common, which does not meet the official requirement to start the profile view from the Initial Approach Fix (IAF). Therefore, the incomplete profile view (which starts from the IF or FAF) requires a waiver from the requirement. By displaying a reduced number of paths on a single page, the vertical profile can start from the correct point in the procedure. This can be seen in the example Modified approach procedure for Boise in Figure 2. The vertical profile for the Current chart shown in Figure 1 starts at the FAF (HOBSI), compared with the Modified chart in Figure 2 which starts at the ICUJY, a common waypoint that is farther out in the procedure.

It should be noted that there are practical disadvantages to separating paths across multiple pages, however. These include having more paper to carry in the flight deck (or more charts to choose from in a database), the need to develop a chart naming convention for each chart in the set, and more time spent searching for the correct page within a set of multiple-page charts. Pilots may be less aware of nearby paths that are not depicted but may be 
available for use. There could be potential communication issues between the flight crews and air traffic controllers about the paths shown on different pages, even with an appropriate naming convention. In addition, there may be increased production costs for the modified charts.

The purpose of this study is to evaluate whether there is sufficient benefit from this de-cluttering technique to warrant additional studies to address the potential drawbacks of the chart modifications. Limitations of this study, discussed below, would also need to be addressed prior to adoption of this decluttering technique.

\section{Experiment Design}

An experiment was conducted to evaluate and compare pilot performance with Current charts versus Modified charts (with fewer paths per procedure). The design of Modified charts and the experiment task and protocol are described in this section.

\section{Modified Charts}

Pilot performance using Modified charts, with a limited number of paths per page, was compared with performance using Current charts. Examples of Current and Modified charts were shown earlier in Figures 1 and 2 for approach procedures and Figures 3 and 4 for departure procedures. Note that all chart figures are shown here at $70 \%$ of their original size, but were shown at full size in the experiment.

FAA Aeronautical Navigation (AeroNav) Products and Jeppesen Sanderson, Inc. ${ }^{1}$, producers of the two most widely used charts in the United States, prototyped examples of Modified charts for a number of airports in coordination with the experimenters. The Modified chart in Figure 1 is an example of the FAA $^{2}$ chart. Each manufacturer modified the charts according to their own standard cartographic conventions.

As noted above, the main modification was to reduce the number of paths per chart. No more than

\footnotetext{
${ }^{1}$ United States government charts are referred to as FAA charts while charts manufactured by Jeppesen Sanderson, Inc., are referred to as Jeppesen charts in this paper.

2 Examples of the Jeppesen charts can be found in Butchibabu and Hansman [5]. They will also be available in a Volpe Center government report in preparation.
}

three paths were included in each modified chart. The paths were grouped so as to maximize the number of common segments. In addition to removing paths that did not share common segments, notes and information for the removed paths were deleted. The white space gained by removing information could have been used to rearrange the remaining information. However this was not done, because we chose to focus only on the effect of the removal of information.

There are eight paths in the Current chart version of the Boise approach to Runway 28L, shown in Figure 1. In the Modified chart version, the procedure was split into four pages with one or two paths per page, grouping paths with common segments. An example of a Modified chart for the BANGS and EMMET IAFs is shown in Figure 2.

Current and Modified departure procedure examples from Salt Lake City are shown in Figures 3 and 4 . There are five paths for the Current departure from Salt Lake City (Figure 3), but only three modified chart pages were created, as paths with common segments were grouped together (Figure 4).

A page naming convention was developed to distinguish between individual pages in the Modified charts. Distinct supplemental names were assigned to each page in addition to the official procedure title. Approach procedures were identified by the IAF names of each path on the Modified chart. Departure procedures were identified by transition fixes or runway names. The names were ordered alphabetically (e.g., 'BANGS/EMETTE' as shown in Figure 2) or sequentially, for runway names (e.g., ' $1 \mathrm{~L} / 1 \mathrm{R} / 7 \mathrm{~L} / 7 \mathrm{R}$ ') within each group.

Each chart manufacturer placed the assigned chart names in different areas of the chart based on their standard cartographic conventions. FAA placed the supplemental chart name under the original title at the top of the page as shown in Figure 2 with the title "BANGS/EMETT" and Figure 4 with the title "ROCK SPRINGS." Jeppesen inserted the supplemental chart name in the plan view for approach procedures and near the graphic description of the route for departure procedures. The same names were assigned to both FAA and Jeppesen charts, regardless of their placement. 


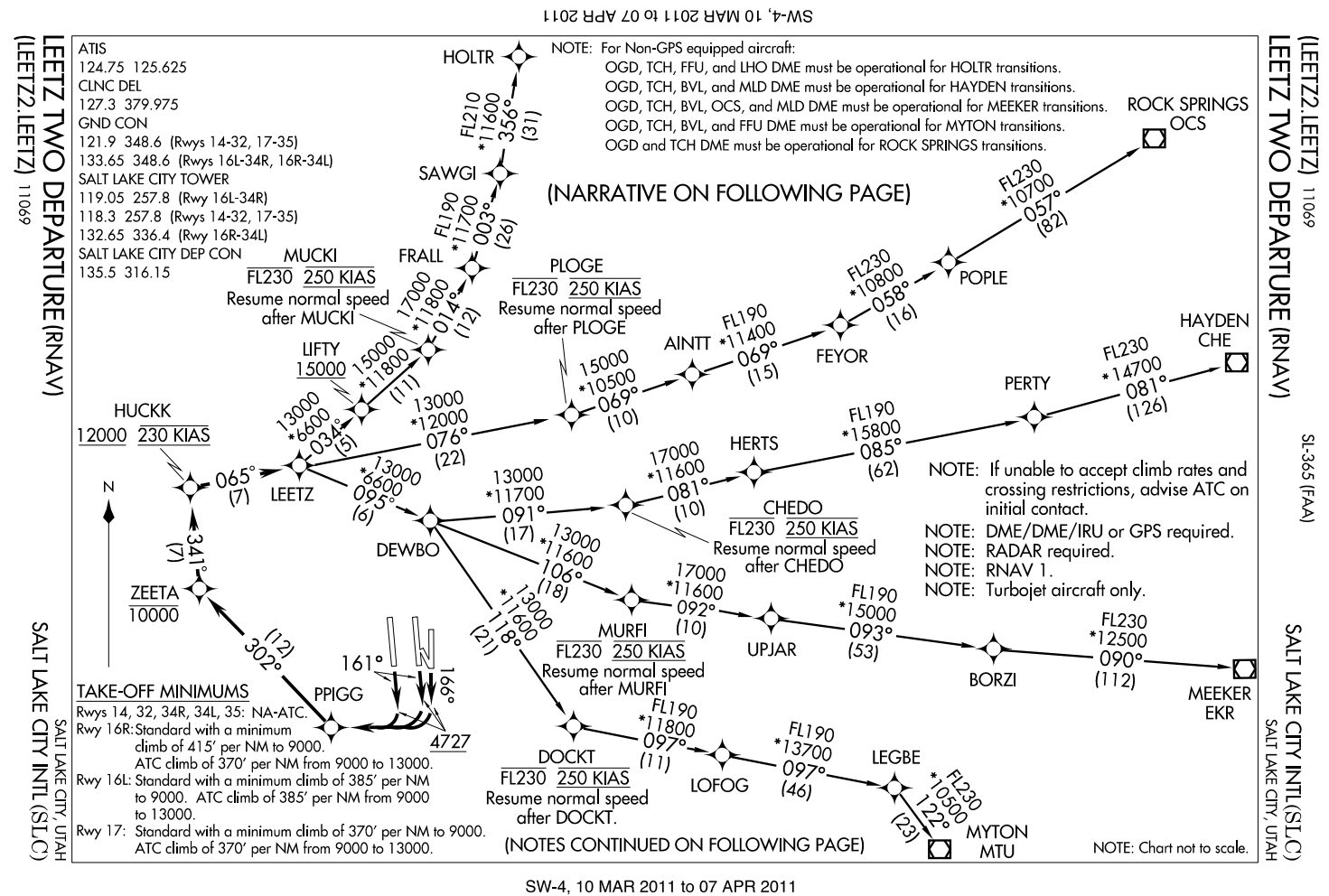

Figure 3. Example Current RNAV Departure Chart

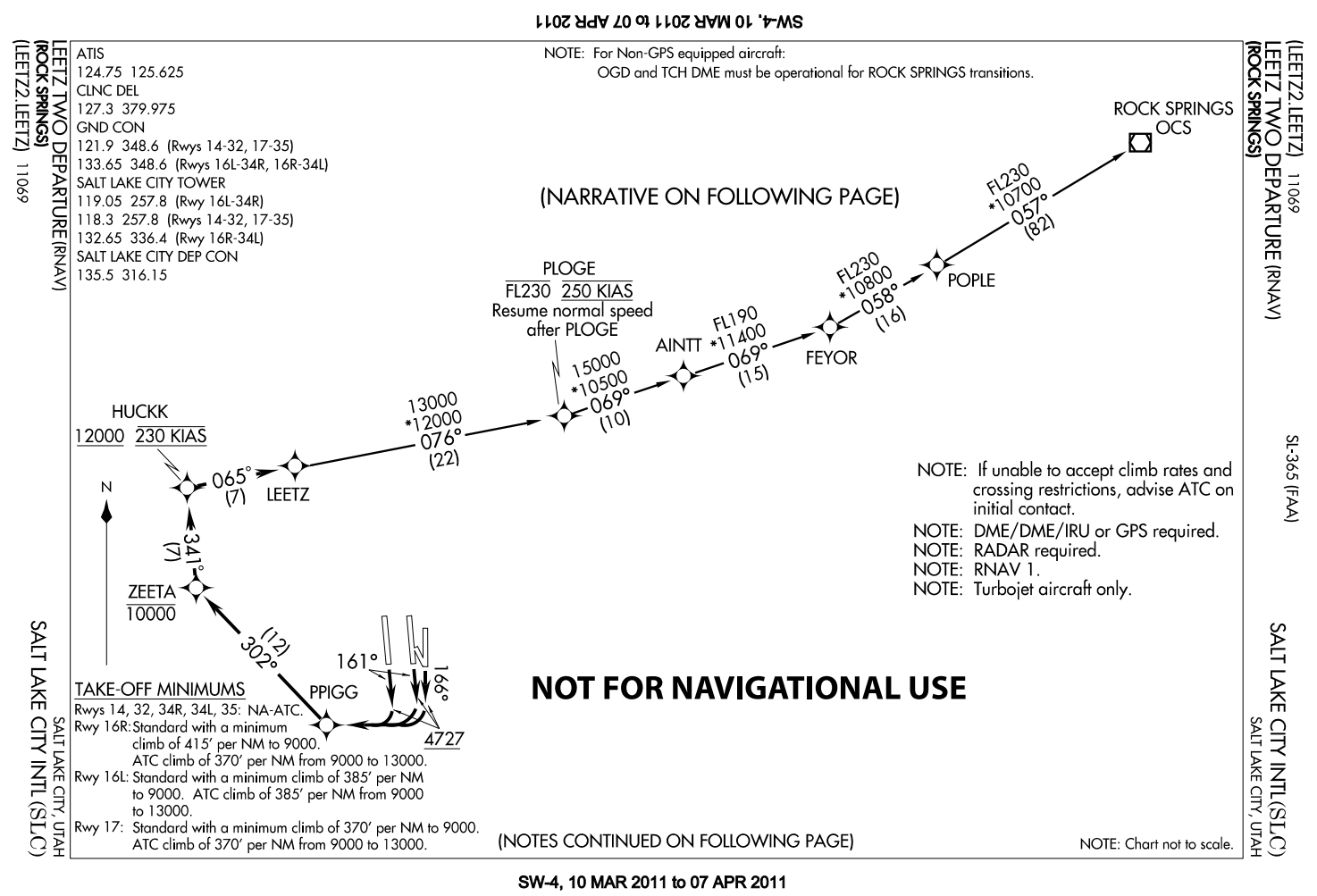

Figure 4. Example Modified RNAV Departure Chart 
In addition to reducing the paths per page on approach procedures and implementing a naming convention, the vertical profile path was extended as described earlier.

A total of six RNAV (RNP) approach and RNAV departure procedures were selected for the study. The charts were selected by subject matter experts for their high level of clutter. Two additional procedures (one RNAV (RNP) approach and one RNAV departure) were used for practice trials in their Current (original) format. Arrival procedures were considered for the study, but not included because they were not as cluttered as the approach and departure procedures.

The three departure procedures modified for the study were from Dallas Fort Worth (DFW), Texas, Salt Lake City (SLC), Utah, and Las Vegas (LAS), Nevada airports. The Modified charts for DFW and LAS each had two pages and the SLC procedure was separated into three pages. The three approach procedures modified for the study were from Boise (BOI), Idaho, Bozeman (BZN), Montana, and Palm Springs (PSP), California airports. The Boise approach procedure was separated into four pages. Bozeman and Palm Springs were each separated into three pages.

\section{Information Retrieval Task}

An information retrieval task was used to determine whether pilot performance improved using Modified charts compared to Current charts. The task required pilots to find a piece of information (e.g., altitude constraint or communication frequency) from a given chart. Information retrieval performance (i.e., time to answer questions and the accuracy of the answers) was measured and compared between Current and Modified charts.

Each trial required pilots to look at one chart in either the Current or Modified format and answer a question associated with that chart. The charts were presented on a high-resolution monitor to optimize visibility. A digital presentation, rather than paper, was used to ensure accurate timing.

Figure 5 shows the electronic display at the beginning of the trial. The pilot was shown a pseudoATC clearance and an information retrieval question before the chart was presented, in order to orient him or herself. An example pseudo ATC clearance question that is presented using Figure 1 or Figure 2 is "You are cleared to Boise Air Terminal (BOI) for the RNAV (RNP) Z RWY 28L via EMETT." An example question associated with this clearance is "What is the distance from ZIZAZ to JADWI?" After reviewing the clearance and question the subject clicked the "Chart" button to show the chart (see Figure 6). At this point the software started a timer to track the amount of time subjects spent looking at the chart. When the subject was ready to answer the question he or she clicked on the "Answer Question" button, which stopped the timer. At this point the chart was grayed out, preventing the pilot from looking at the chart (see Figure 7). The pilot would then click on the "Answer Question" button again and type in their answer (3.1NM in this example). If the pilot forgot their answer and wanted to view the chart again, he or she could click on "Chart" to call up the chart again; this action restarts the timer. The information retrieval time was the cumulative time the chart was visible to the subject.

Responses to questions were recorded and scored for accuracy. The response times and accuracy for each chart type (Current and Modified) and procedure type (approaches and departures) were evaluated separately.

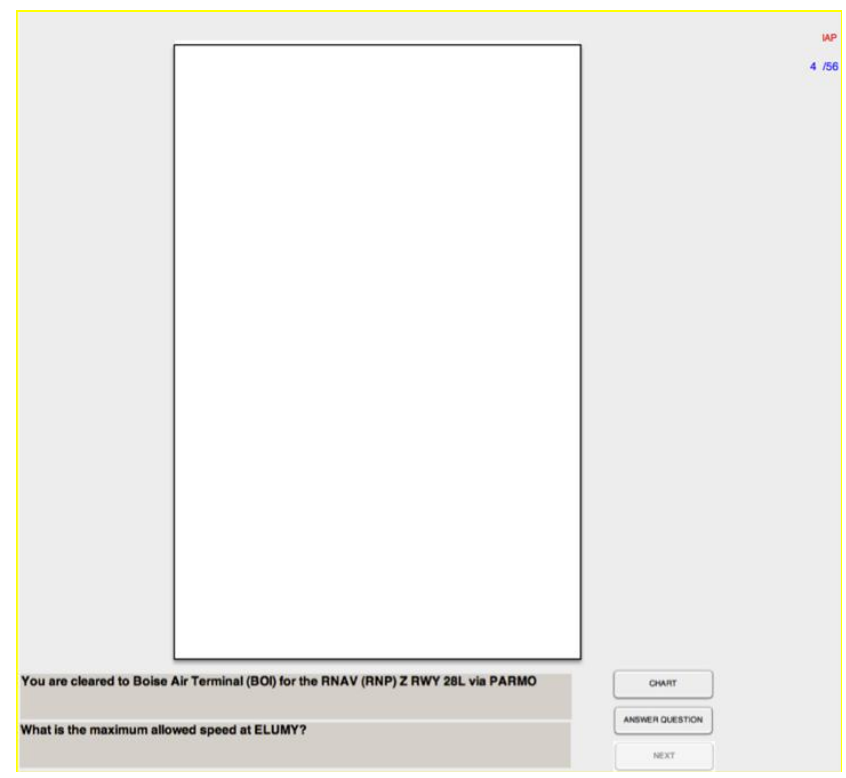

Figure 5. Display at the beginning of trial.

\section{Experiment Protocol}

Prior to beginning the experiment, each pilot was given the option of using the chart type with which they were most familiar (Jeppesen or FAA). All 47 pilots were comfortable with Jeppesen charts. 
To evaluate the FAA charts, the experimenters requested that 14 pilots (eight Airline and six Corporate) use FAA charts instead of Jeppesen. To familiarize these pilots with FAA charts, the experimenters developed and administered a 10 minute FAA chart refresher course prior to the start of the experiment.

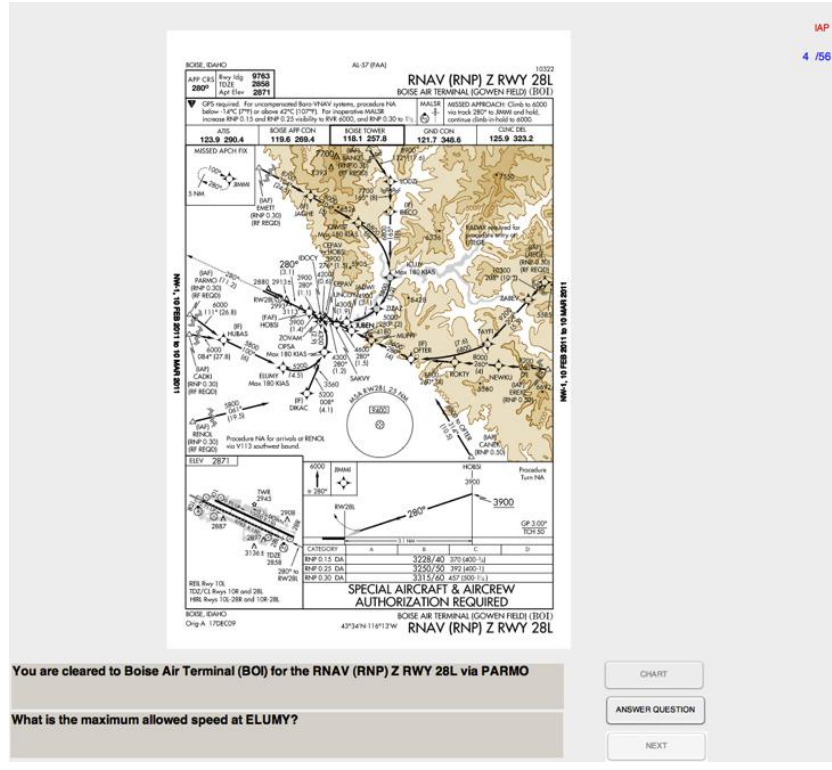

Figure 6. Display after "Chart" button is clicked.

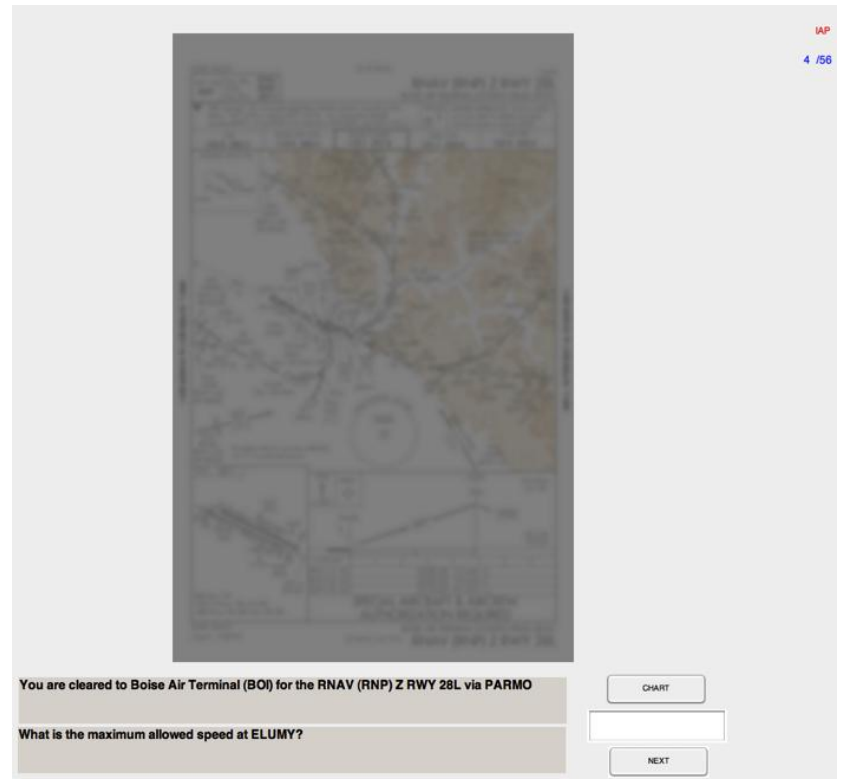

Figure 7. Display after "Answer Question" is clicked.

Figure 8 shows a flow diagram of the experimental protocol. The experiment took approximately one hour, plus ten minutes for participants who took the FAA chart refresher course.

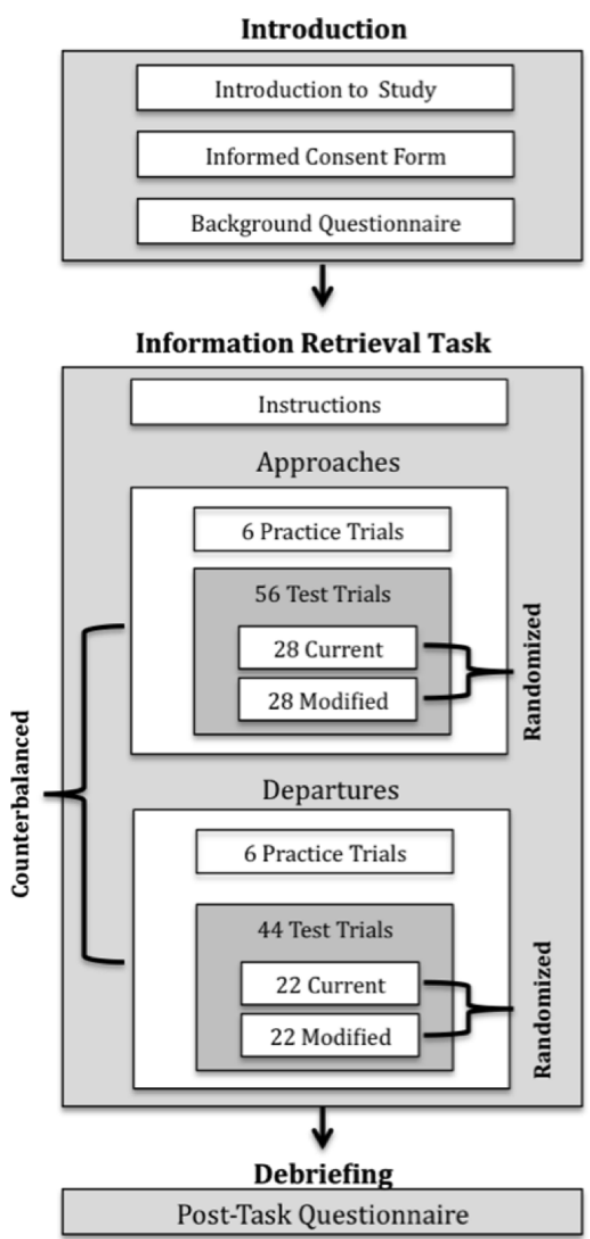

Figure 8. Flow Diagram of the Study Protocol.

First, each participant was given an introduction to the study, informed consent form, and background questionnaire. The introduction summarized the purpose and potential outcomes of the study. The background questionnaire covered each subject's familiarity with RNAV and RNP procedures. Other relevant information about their flight experience was also recorded.

Written instructions for the information retrieval task were then provided to the subjects. The instructions told the pilots to respond to the questions as quickly and as accurately as possible. The task was divided into two blocks separated by a rest period. One block was for approaches and the other block was for departures. The order of the two blocks was counterbalanced across subjects. The approach block contained 56 test trials and the departure block contained 44 test trials. Each test trial consisted of answering one question for a specific chart. Within 
each block, the two chart formats (Current and Modified) were presented in random order. Half of the questions in each block pertained to the Current charts while the other half pertained to the Modified charts. Chart format was a within-subjects variable, meaning that each participant answered questions about both Current and Modified charts.

The study concluded with a post-task questionnaire where pilots provided information on their experience with the procedures tested in the experiment. Pilots were also asked to provide feedback about the experiment.

\section{Experiment Results and Discussion}

\section{Participants}

A total of 47 pilots participated in the study. Participants were professional pilots from corporate or airline operators in the United States. All subjects were current and licensed instrument-rated, RNPqualified pilots, meaning they were trained to meet the Authorization Requirement (AR) to fly these procedures [8], [9]. Based on the background questionnaire presented to the pilots, the flight time for participants ranged between 2,200 hours and 24,000 hours, with an average of 11,484 and a median of 10,250 hours. See Table 1 for details regarding pilot experience and background. Note that seven airline instructor pilots from an airplane manufacturing company were included in the Airline Pilot group. Pilots were not compensated for their participation.

All pilots had received simulator training on RNAV procedures within the last 12 months. Table 1 shows the average number of RNAV (RNP) IAP and RNAV SID procedures flown in the last active month, according to the pilots' responses to the background questionnaire.

Prior to beginning the experiment, pilots rated their comfort levels with RNAV departure procedures and RNAV (RNP) approach procedures. In general, pilots recorded high comfort levels with RNAV departure and RNAV (RNP) approach procedures. For approaches, 34 pilots $(72 \%)$ rated their comfort level as 4 or 5 , on a scale from 1 (least comfortable) to 5 (most comfortable). For departures, 33 pilots $(70 \%)$ rated their comfort level as 4 or 5 . Out of the 47 pilots, three had never flown RNAV (RNP) approaches in line operations.
Table 2 summarizes the pilot experience over the past 12 months at airports from which the procedures for the study were selected, based on the post-task questionnaire completed by pilots. In general, pilots had the least experience at Boise and Bozeman, and the most experience at Las Vegas, Palm Springs, and Dallas-Fort Worth.

According to the post-task questionnaire, all participants agreed that information retrieval questions and charts were reasonably presented and the experiment display was easy to understand.

\section{Accuracy}

Each pilot was graded on 98 information retrieval questions. ${ }^{3}$ In general, pilots answered questions correctly. Average accuracy across all 47 participants was 99.5\%; 34 pilots answered all 98 questions correctly. The lowest score was $94.9 \%$ where the pilot missed five out of the 98 questions. There were no significant differences in accuracy between Current and Modified charts for IAPs or SIDs. Also, no differences in accuracy were found between chart manufacturer (FAA and Jeppesen) or pilot type (Airline and Corporate).

\section{Response Time}

Figure 9 presents average response times for Current and Modified charts by procedure type. The average response time for pilots using Modified charts was significantly faster than for pilots using Current charts, for both types of procedures (see Table 3). For approaches, the mean response time was 16.87 seconds for Current charts and 10.66 seconds for Modified charts. For departures, the mean response time was 16.19 seconds for Current charts and 13.26 seconds for Modified charts. That is, the mean response time for approach procedures was reduced by 6.2 seconds and for departure procedures by 2.9 seconds. The error bars in Figure 9 depict standard error, which was less than one second in all cases. Two-tailed paired t-tests were conducted on the logarithm of response times of Current and Modified charts to account for the skew in the data.

\footnotetext{
${ }^{3}$ Data from two questions out of the 100 in the study were excluded due to a spelling error.
} 


\section{Effect of Specific Airport/Procedure}

Figure 10 presents average response times for each of the six airports in the study. Results for all airports are consistent with the overall response pattern, in that pilots performed significantly faster using Modified charts than using Current charts, regardless of procedure.

Results from a two-tailed paired samples t-test conducted on the logarithm of response times are displayed in Table 4. All procedures show statistically significant improvements in response times for Modified charts compared to Current charts. This effect was observed for both approach and departure procedures.

\section{Limitations of the Study}

There are a number of limitations to this study. First, the charts selected for the study were specifically chosen for their high clutter levels. Thus, the applicability of the technique to less cluttered charts has yet to be evaluated. Further testing would be required to determine criteria for implementing the modifications. That is, chart manufacturers will need specific guidance to decide when or when not to use the technique; over use of the technique has the potential to impede pilot performance due to the numerous drawbacks mentioned earlier.

A second limitation of the study is that it did not address the potential for increased times to retrieve the correct page in an operational setting. That is, the task of retrieving a particular chart was not included in this study. A well-designed chart naming convention could potentially mitigate this concern, but the convention would have to be an accepted industry standard.

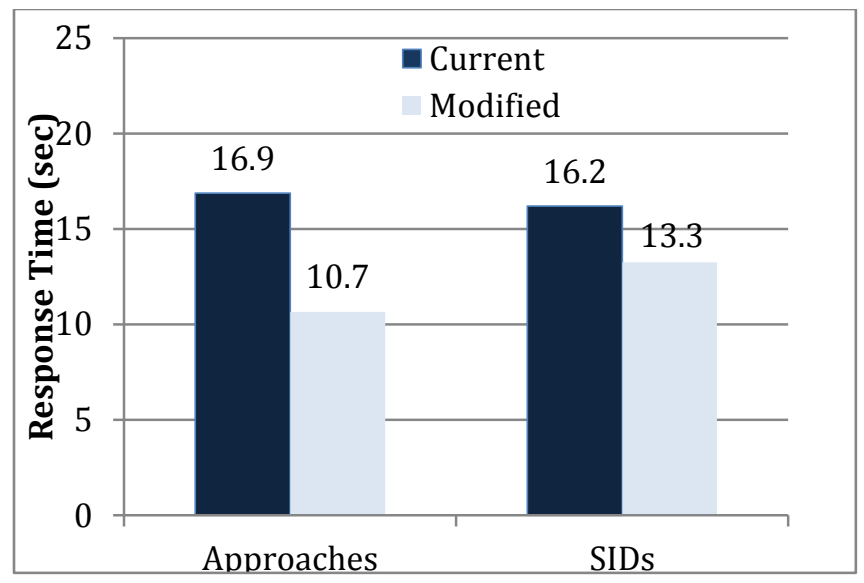

Figure 9. Average Response Times by Procedure Type

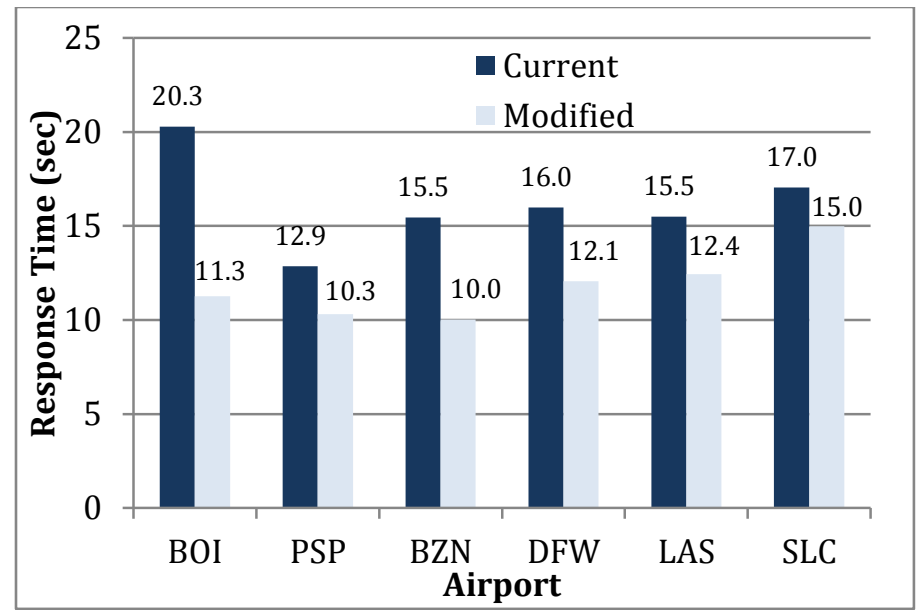

Figure 10. Average Response Times by Airport

Table 1. Participant Flight Experience

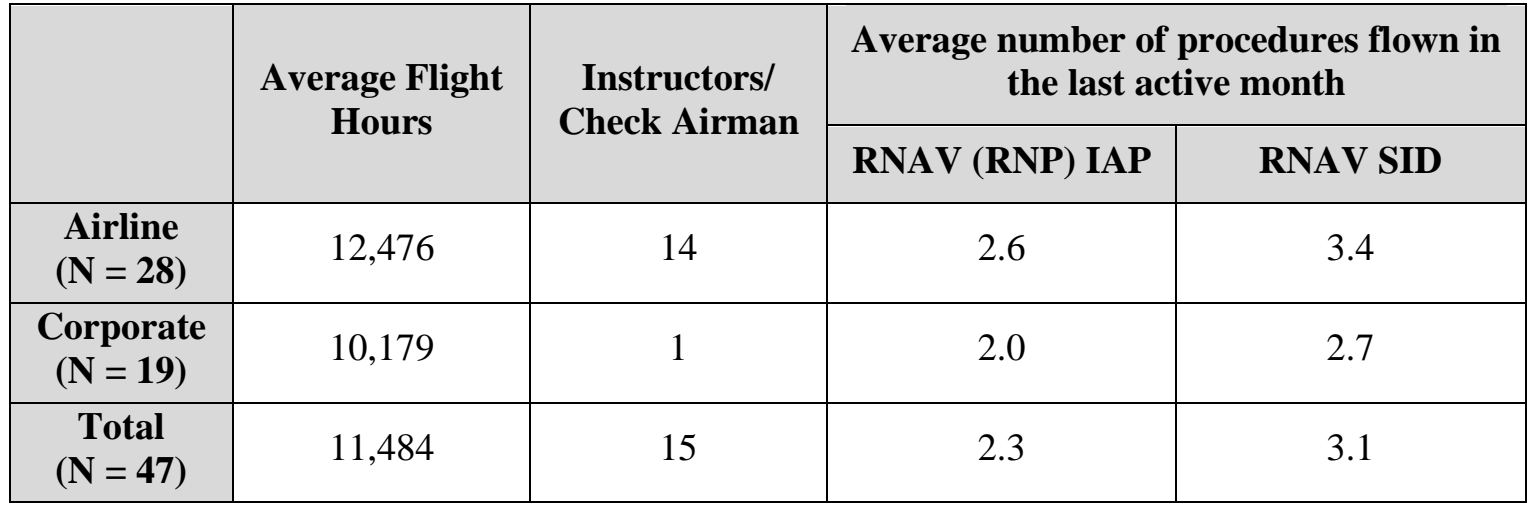


Table 2. Participant experience at airports in the study.

\begin{tabular}{|c|c|c|c|c|}
\hline & Airport & $\begin{array}{l}\text { Corporate } \\
(\mathrm{N}=19)\end{array}$ & $\begin{array}{r}\text { Airline } \\
(\mathrm{N}=28)\end{array}$ & $\begin{array}{r}\text { Total } \\
(\mathrm{N}=47)\end{array}$ \\
\hline \multirow{3}{*}{$\begin{array}{l}\quad \text { RNAV } \\
\text { (RNP) } \\
\text { Approaches }\end{array}$} & Boise, Idaho & 1 & 0 & 1 \\
\hline & $\begin{array}{l}\text { Bozeman, } \\
\text { Montana }\end{array}$ & 0 & 0 & 0 \\
\hline & $\begin{array}{r}\text { Palm } \\
\text { California }\end{array}$ & 2 & 13 & 15 \\
\hline \multirow{3}{*}{$\begin{array}{l}\text { RNAV } \\
\text { SIDs }\end{array}$} & $\begin{array}{l}\text { Dallas-Fort Worth, } \\
\text { Texas }\end{array}$ & 2 & 10 & 12 \\
\hline & $\begin{array}{l}\text { Las } \quad \text { Vegas, } \\
\text { Nevada }\end{array}$ & 15 & 7 & 22 \\
\hline & $\begin{array}{l}\text { Salt Lake City, } \\
\text { Utah }\end{array}$ & 5 & 1 & 6 \\
\hline
\end{tabular}

Table 3. Results by Procedure Type.

\begin{tabular}{|l|c|c|c|}
\hline & $\begin{array}{c}\text { Current } \\
\text { (Seconds) }\end{array}$ & $\begin{array}{c}\text { Modified } \\
\text { (Seconds) }\end{array}$ & Statistical Test \\
\hline Approaches & 16.87 & 10.66 & $t_{46}=16.4, p<0.01$ \\
\hline Departures & 16.19 & 13.26 & $t_{46}=6.7, p<0.01$ \\
\hline
\end{tabular}

Table 4. Results by Airport

\begin{tabular}{|l|c|c|c|}
\hline $\begin{array}{c}\text { Airport } \\
\text { Code }\end{array}$ & $\begin{array}{c}\text { Mean Response Time } \\
\text { for Current Charts } \\
\text { (seconds) }\end{array}$ & $\begin{array}{c}\text { Mean Response Time } \\
\text { for Modified Charts } \\
\text { (seconds) }\end{array}$ & Statistical Comparison \\
\hline BOI & 20.29 & 11.27 & $t_{46}=14.1, p<0.001$ \\
\hline BZN & 15.45 & 10.02 & $t_{46}=10.0, p<0.001$ \\
\hline PSP & 12.85 & 10.32 & $t_{46}=4.6, p<0.001$ \\
\hline DFW & 15.97 & 12.05 & $t_{46}=4.2, p<0.001$ \\
\hline LAS & 15.49 & 12.43 & $t_{46}=3.7, p=0.001$ \\
\hline SLC & 17.05 & 14.98 & $t_{46}=3.4, p=0.001$ \\
\hline
\end{tabular}


Another limitation is that the study was designed to determine the benefit of just one isolated factor: separating paths across multiple pages to reduce the number of paths shown per page. A logical next step is to zoom and re-center the charts for optimal use of the space available. However, these techniques may, or may not, further improve information retrieval performance. Evaluations are needed to ensure that zooming and re-centering do not impede performance in unexpected ways. For example, it may be difficult to orient oneself across chart pages if they are at different scales with different centers. This could be an issue if the pilot is asked to maneuver from a flight path on one page to a flight page on a different page.

Another potential limitation of the study was that we used a high-resolution electronic display presentation instead of testing with paper charts for fidelity. The computer was used so that more accurate response time could be achieved. A paperbased display might have provided higher face validity for the paper charts. However, the focus of the experiment was on chart format and understanding the benefits of the de-cluttering technique, which are expected to be independent of paper or electronic display format. Therefore, the use of a computer monitor for the study was not in itself a limitation of the study. However, a different study could have been designed around the use of paper charts. That study would have to consider a variety of other factors, such as how to ensure accurate timing, and the practical constraints of paper charts (e.g., bound versus loose, size of the paper, etc.)

\section{Summary and Conclusion}

A de-cluttering technique was evaluated to investigate potential improvements in chart usability for RNAV and RNAV (RNP) charts. The modification technique involved separating flight paths across multiple pages to reduce the number of paths depicted on one page. The experiment was an information retrieval task, in which each pilot answered questions based on a given chart. Pilot performance was analyzed in terms of the time and accuracy of the responses to each question.

Results show that the de-cluttering technique significantly reduced pilot response times on the information retrieval task when using Modified (i.e., de-cluttered) charts compared to Current charts. This reduction in response time for Modified charts was consistent across the procedures (approaches and departures), chart manufacturers (FAA and Jeppesen), pilot types (Airline and Corporate), and all six airports that were included in this study. The average reduction in response time was just over 6 seconds for approach procedures and approximately 3 seconds for departure procedures. In critical phases of flight where these procedures are typically flown, this decreased response time suggests there may be benefits in using charts with fewer paths per page. Though there are several drawbacks to this modification technique that must be explored before implementation, the results of the experiment are potentially useful for future design of paper charts, as well as for the design of data-driven electronic charts.

\section{References}

[1] MITRE Center for Advanced Aviation Syst. Develop. (2011). Performance Based Navigation Capabilities Report. [Online]. Available: http://www.mitrecaasd.org/PBNCapabilityReport/

[2] R. Barhydt and C. Adams, "Human Factors Considerations for Area Navigation Departure and Arrival Procedures," presented at the 25th Congr. of the Int. Council of the Aeronautical Sci, Hamburg, Germany, Sep. 3-8. 2006. [Paper] Available: http://ntrs.nasa.gov/.

[3] R. Barhydt and C. Adams, "Human Factors Considerations for Performance-Based Navigation," NASA Langley, Langley, VA, Tech. Rep. NASA/TM-2006-21453, Dec. 2006. Available: http://ntrs.nasa.gov/.

[4] A. Butchibabu, A. Midkiff, A. Kendra, R. J. Hansman, D. Chandra, "Analysis of safety reports involving area navigation and required navigation performance procedures," in Proc. Int. Conf. HumanComputer Interaction in Aeronautics, Cape Canaveral, FL, 2010. Available: http://ntl.bts.gov/lib/44000/44100/44160/hciaero_1_. pdf.

[5] A. Butchibabu and R. J. Hansman. (2012, May). Evaluating the Depiction of Complex RNAV/RNP Procedures and Analyzing a Potential De-Cluttering Technique. Int. Center for Air Transportation, Massachusetts Institute of Technology, Cambridge, MA. [Online]. Available: 
http://web.mit.edu/abhiznab/Public/ICAT\%20Report _Butchibabu.pdf.

[6] D. Chandra, R. Grayhem, and A. Butchibabu (in press). "Area Navigation and Required Navigation Performance Procedures and Depiction," Volpe Nat. Transportation Syst. Center, U.S. DOT, Cambridge, MA, Tech. Rep. DOT/FAA/TC-12/8.

[7] Aeronautical Charting Forum. (2011, Oct.). \#0902-220, Recommendation Document FAA Control. [Online]. Available: http://aeronav.faa.gov/index.asp?xml=aeronav/acf.

[8] Federal Aviation Administration. (2011, Feb.). AC 90-101A, Approval Guidance for RNP Procedures with AR. Available: http://www.faa.gov/documentLibrary/media/Advisor y_Circular/AC\%2090-101A.pdf.

[9] Federal Aviation Administration. (2007, Mar.). AC 90-100A, U.S. Terminal and En Route Area Navigation (RNAV) Operations. Available: http://rgl.faa.gov/Regulatory_and_Guidance_Library/ rgAdvisoryCircular.nsf/0/5c94e4f44ba319a98625729 c00612f37/\$FILE/AC\%2090-100A.pdf.

\section{Acknowledgements}

This paper was prepared by MIT and the Aviation Human Factors Division at the Volpe National Transportation Systems Center. MIT was funded by the Volpe Center (Contract No. DTR5707-D-30006). The Volpe Center was funded by the FAA Human Factors Research and Engineering Group (ANG-C1) in support of Aviation Safety (AVS).
The authors would like to thank Andrew Kendra (Volpe Center), Alan Midkiff (MIT) and Alan Yost (Volpe Center) for their useful insights on the study, particularly with the experiment design and subject recruitment.

Valuable support was received from the FAA program managers, Tom McCloy and Dan Herschler, and our FAA technical sponsors, Kathy Abbott and Mark Steinbicker. The instrument procedure charts presented in this paper were developed in collaboration with FAA AeroNav Products: John Moore, Valerie Watson, Brad Rush, and Alex Rushton, and Jeppesen: Ted Thompson, and Jeff Williams.

Particular thanks go to Maura Lohrenz (Volpe Center) for her valuable feedback on this paper.

Assistance in subject recruitment was received from Air Line Pilots Association (ALPA) and National Business Aviation Association (NBAA). Numerous professional pilots' participation in the study, and their employers' support, greatly contributed to this work.

The views expressed herein are those of the authors and do not necessarily reflect the views of the Volpe National Transportation Systems Center, the Research and Innovative Technology Administration, or the United States Department of Transportation.

\section{1st Digital Avionics Systems Conference October 14-18, 2012}

